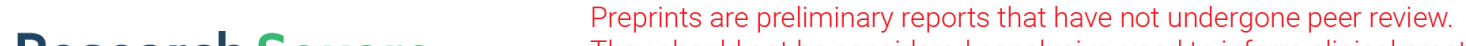 They should not be considered conclusive, used to inform clinical practice, or referenced by the media as validated information. \\ Clinical evaluation of a multiplex RT-PCR assay for detection of SARS-CoV-2 in individual and pooled upper respiratory tract samples
}

\section{Melissa Laverack}

Cornell University College of Veterinary Medicine

Rebecca L. Tallmadge

Cornell University College of Veterinary Medicine

Roopa Venugopalan

Cornell University College of Veterinary Medicine

Brittany Cronk

Cornell University College of Veterinary Medicine

XiuLin Zhang

Cornell University College of Veterinary Medicine

Rolf Rauh

Tetracore Inc

\section{Amy Saunders}

Tetracore Inc

William M. Nelson

Tetracore Inc

Elizabeth Plocharczyk

Cayuga Medical Center

Diego G. Diel ( $\nabla$ dgdiel@cornell.edu )

Cornell University College of Veterinary Medicine https://orcid.org/0000-0003-3237-8940

\section{Research Article}

Keywords: Nasopharyngeal (NP), anterior nares (AN), sensitive, high-throughput, cost-effective

Posted Date: March 4th, 2021

DOI: https://doi.org/10.21203/rs.3.rs-280275/v1

License: (c) (i) This work is licensed under a Creative Commons Attribution 4.0 International License.

Read Full License 
Version of Record: A version of this preprint was published at Archives of Virology on July 14th, 2021. See the published version at https://doi.org/10.1007/s00705-021-05148-1. 


\section{Abstract}

The aim of this study was to identify and validate a sensitive, high-throughput and cost-effective SARSCoV-2 RT-PCR assay to be used as a surveillance and diagnostic tool for SARS-CoV-2 in a University surveillance program. We conducted a side-by-side clinical evaluation of a newly developed SARS-CoV-2 multiplex assay (EZ-SARS-CoV-2 Real-Time RT-PCR) with the commercial TaqPath COVID-19 Combo kit, which has an Emergency Use Authorization from the FDA. The EZ-SARS-CoV-2 RT-PCR incorporates two assays targeting the SARS-CoV-2 $\mathrm{N}$ gene, an internal control targeting the human RNase $\mathrm{P}$ gene, and a PCR inhibition control in a single reaction. Nasopharyngeal (NP) and anterior nares (AN) swabs were tested as individuals and pools with both assays and in the ABI 7500 Fast and the QuantStudio 5 detection platforms. The EZ-SARS-CoV-2 RT-PCR assay analytical sensitivity was $250 \mathrm{copies} / \mathrm{ml}$ or approximately 1.75 genome copy equivalents per reaction. Clinical performance of the EZ-SARS-CoV-2 assay was determined using NP and AN samples tested in other laboratories. The diagnostic sensitivity of the assay ranged between 94 and $96 \%$ across the detection platforms, and the diagnostic specificity was $94.06 \%$. The positive predictive value was $94 \%$ and the negative predictive value ranged from 94 to $96 \%$. Pooling five NP or AN specimens yielded $93 \%$ diagnostic sensitivity. The overall agreement between these SARS-CoV-2 RT-PCR assays was high, supported by Cohen's kappa value of 0.93 . The EZ-SARSCoV-2 RT-PCR assay performance attributes of high sensitivity, excellent performance in AN sample matrix and in pooled upper respiratory samples support its use in a high-throughput surveillance testing program.

\section{Introduction}

The Coronavirus Disease 19 (COVID-19) pandemic caused by severe acute respiratory syndrome coronavirus 2 (SARS-CoV-2) became a public health emergency due to the rapid and widespread dissemination of the virus at a global scale [1-3]. Rapid and accurate diagnosis of SARS-CoV-2 infection are critical to prevent virus transmission through isolation of positive patients and implementation of quarantine to contact persons and to provide adequate treatment to clinically affected patients [4]. Given that SARS-CoV-2 causes asymptomatic infections [5-7], and that it can be effectively transmitted prior to the development of symptoms [8-11], widespread testing of symptomatic and asymptomatic populations is essential for effective disease control. To accomplish this, highly sensitive, accurate and efficient tests and testing workflows are needed. This is especially important, given the high demand for SARS-CoV-2 testing and the resultant supply shortages that limit the number of SARS-CoV-2 and other diagnostic assays that can be performed, as reported by the American Society for Microbiology [12].

SARS-CoV-2 is a newly emerging member of the genus Sarbecovirus within the family Coronaviridae [13]. The SARS-CoV-2 genome consists of a single-stranded RNA molecule of approximately $30 \mathrm{~kb}$ in length [14]. Therefore, assays for detection of SARS-CoV-2 are often based on real-time reverse transcriptase polymerase chain reaction (RT-PCR) $[15,16]$ and many RT-PCR assays have been given Emergency Use Authorization by the U.S. Food and Drug Administration [17]. The high analytical sensitivity of RT-PCR allows its use for pooled testing, in which multiple samples are mixed and tested in a single reaction, thus 
increasing testing efficiency and significantly lowering the costs [18]. The feasibility of pooled testing for detection SARS-CoV-2 has been demonstrated in multiple studies [19-22].

We were tasked with identifying a sensitive SARS-CoV-2 assay that could be performed in an efficient manner and provide sensitive and specific surveillance and diagnostic testing capability to support Cornell University's surveillance program and campus re-opening in the fall semester of 2020. The present study was undertaken to identify and validate a SARS-CoV-2 multiplex RT-PCR assay that could support high throughout and cost-effective testing with rapid turn-around time. We evaluated the clinical performance of a newly developed SARS-CoV-2 multiplex RT-PCR assay (targeting two regions of the SARS-CoV-2 N gene, in addition to an internal control targeting the human RNase P gene, and a PCR inhibition control) and compared it to the performance of a commercial assay with an EUA from the FDA. To facilitate efficient testing, increase testing capacity, and decrease overall testing costs of the University surveillance program, assay performance was also evaluated on pooled samples.

\section{Materials And Methods}

Clinical specimens. De-identified frozen specimens were shared with the Cornell COVID-19 Testing Laboratory (CCTL) by other COVID-19 testing laboratories in the United States. A total of 201 nasopharyngeal (NP) swabs and 24 anterior nares (AN) swabs were included in this study. Sixty NP swabs were tested in the originating laboratory with the Xpert ${ }^{\circledR}$ Xpress SARS-CoV-2 assay (Cepheid, Sunnyvale, CA) and of these, 30 had SARS-CoV-2 positive results and 30 had SARS-CoV-2 negative results. The other 141 NP swabs were tested in the originating laboratory with the TaqPath COVID-19 Combo Kit Multiplex Real-Time RT-PCR assay (Thermo Fisher Scientific Inc., Waltham, MA); 70 were SARS-CoV-2 positive and 71 were determined as SARS-CoV-2 negative. The AN swabs were originally tested with the New York State Department of Health (NYSDOH) SARS-CoV-2 RT-PCR assay, 12 had SARS-CoV-2 positive results and 12 were determined as negative.

Sample pooling. Pool sizes of 5 or 10 samples were contrived from one SARS-CoV-2 positive sample combined with 4 or 9 negative samples, respectively. Two hundred $\mu$ of each sample were pooled. Twenty individual positive NP swab samples with original Ct values of 28 or greater were selected to generate the pools. Twelve individual positive AN swab samples with original $\mathrm{Ct}$ values between 19 and 34 were selected to generate the pools.

Nucleic acid extraction. Nucleic acid was extracted from $200 \mathrm{ml}$ of each individual swab supernatant or pooled samples using the MagMAX Viral/Pathogen II Nucleic Acid Isolation Kit (Applied Biosystems, Foster City, CA) and the KingFisher Flex Magnetic Particle Processor following the manufacturer's procedures (Thermo Fisher Scientific Inc.). Prior to extraction each sample was vortexed at $100 \times \mathrm{g}$ for 10 sec. Nucleic acid was eluted in $50 \mathrm{ml}$ of elution buffer and used for SARS-CoV-2 detection as described below.

SARS-CoV-2 RT-PCR assays. Two assays for SARS-CoV-2 real-time RT-PCR were used in this study, the newly developed EZ-SARS-CoV-2 Real Time RT-PCR assay (Tetracore, Inc., Rockville, MD) and the TaqPath 
COVID-19 Combo Kit Multiplex Real-Time RT-PCR assay (Thermo Fisher Scientific Inc.). The EZ-SARSCoV-2 assay was evaluated and compared to the TaqPath COVID-19 Combo Kit Multiplex Real-Time RTPCR assay which served as a reference standard assay with a current EUA from FDA. Negative extraction, negative amplification, and positive amplification controls were included on each assay run. RT-PCR assays were performed in two detection systems: the ABI 7500 Fast Real-Time PCR System (with SDS v1.5.1 software, Applied Biosystems, Waltham, MA) and the QuantStudio 5 Real-Time PCR System (with QuantStudio Design and Analysis Desktop Software v1.5.1, Applied Biosystems).

The EZ-SARS-CoV-2 Real-Time RT-PCR (Tetracore, Inc.) is a multiplex assay targeting the SARS-CoV-2 N gene (two target regions, with both probes labeled with FAM reporter dye), the human RNase P gene (CY5 reporter dye), and an inhibition control (IC, TAMRA reporter dye). The amplification cycling conditions were $48^{\circ} \mathrm{C}$ for 15 minutes; $95^{\circ} \mathrm{C}$ for 2 minutes; 45 cycles of $95^{\circ} \mathrm{C}$ for 10 seconds, $60^{\circ} \mathrm{C}$ for 40 seconds using $18 \mathrm{ml}$ of combined reagents and $7 \mathrm{ml}$ of nucleic acid template. RT-PCR data was analyzed for SARS-CoV-2 and IC targets by setting the threshold at $3 \%$ of the final normalized fluorescence of the positive amplification control. For the human RNase $\mathrm{P}$ gene, the threshold was set at $3 \%$ of the approximate average of the final normalized fluorescence of all samples in a single plate run. The baseline was set automatically by the software for all reporter dyes.

The TaqPath COVID-19 Combo Kit Multiplex Real-Time RT-PCR assay (Thermo Fisher Scientific Inc.) targets three regions of the SARS-CoV-2 genome (ORF1ab, N, and S genes with FAM, VIC, and ABY reporter dyes, respectively) and an inhibition control target (MS2 bacteriophage, JUN reporter dye). The amplification cycling conditions were $25^{\circ} \mathrm{C}$ for 2 minutes; $53^{\circ} \mathrm{C}$ for 10 minutes; $95^{\circ} \mathrm{C}$ for 2 minutes; 40 cycles of $95^{\circ} \mathrm{C}$ for 3 seconds, $60^{\circ} \mathrm{C}$ for 30 seconds using $15 \mathrm{ml}$ of combined reagents and $10 \mathrm{ml}$ of template. RT-PCR data was analyzed for SARS-CoV-2 targets by setting the threshold at $10 \%$ of the final normalized fluorescence of the positive amplification control. For MS2, the threshold was set at $10 \%$ of the approximate average of the final normalized fluorescence of all samples in a single plate run. The baseline was set automatically by the software for all reporter dyes. For result interpretation, TaqPath RTPCR data was analyzed with auto settings and imported to the Applied Biosystems COVID-19 Interpretive Software SOP version 1.2.

Analytical sensitivity. The limit of detection (LoD) of the EZ-SARS-CoV-2 RT-PCR was determined by preparing two-fold serial dilutions in VTM and AN sample matrix ranging from 1,000 to $62.5 \mathrm{copies} / \mathrm{ml}$ of a commercial standard containing the full length SARS-CoV-2 genomic RNA (AccuPlex SARS-CoV-2 Verification Panel - Full Genome, Seracare Life Sciences, Inc., Milford, MA, catalog number 0505-0168). Each serial dilution was tested three times independently using the ABI 7500 Fast and the QuantStudio 5 detection systems. A preliminary LoD was defined as the lowest concentration in which $100 \%$ of the replicates were positive. The final LoD of the assay was determined by testing 20 replicates containing 250 and 500 viral genome copies/ml and the LoD was defined as the lowest concentration in which at least $19 / 20(95 \%)$ of the replicates were detected. 
Analytical specificity. Initial cross-reactivity analyses of the EZ-SARS-CoV-2 RT-PCR primers and probes were performed in silico. Primer and probe sequences were blasted against public domain nucleotide sequences. The database search parameters were as follows: 1) The nucleotide collection consisted of GenBank NT and RefSeq sequences, but excluded EST, STS, GSS, WGS, TSA, and patent; 2) The database was non-redundant. Identical sequences were merged into one entry, while preserving the accession, $\mathrm{GI}$, title and taxonomy information for each entry; 3 ) Database was updated on 02/11/2020; 4) The search parameters automatically adjusted for short input sequences and threshold was set at 1000;5) The match and mismatch scores were 1 and -3 , respectively. Additionally, Needleman-Wunsch alignments [23] were performed against a defined set of sequences in Supplementary Table 1. Each primer and probe of the EZ-SARS-CoV-2 PCR assay was aligned to the sequences listed in Supplementary Table 1. No gaps were allowed in the alignment and a match matrix was used. The matrix scored the alignment with a 1 for match and a 0 for anything else. The alignment score was the number of matches between the primer or probe and the pathogen. The frequency of the alignment was the number of matches divided by the length of the primer or probe.

To exclude cross-reactivity of the EZ-SARS-CoV-2 RT-PCR assay against other human pathogens and confirm its specificity in the wet test condition, the assay was tested against 40 non-target organisms known to infect humans (Supplementary Table 2). Nucleic acid from each organism (concentration of $>$ $10^{6} \mathrm{CFU} / \mathrm{ml}$ or $>10^{4} \mathrm{TCID}_{50} / \mathrm{ml}$, when available from the vendor) was extracted using the Qiagen QIAamp Viral RNA Mini Kit and subjected to real-time PCR using the EZ-SARS-CoV-2 assay on the ABI 7500 Fast real-time PCR System. Each pathogen was tested in triplicate. SARS-CoV-2 positive and negative controls were included in each assay run.

Reproducibility. The intra- and inter-run reproducibility of the EZ-SARS-CoV-2 PCR assay were evaluated. For this, 10-fold serial dilutions containing between 100 and 10,000 genome copies/ml of SARS-CoV-2 (AccuPlex SARS-CoV-2 Verification Panel - Full Genome, Seracare Life Sciences, Inc., catalog number 0505-0168) were prepared in viral transport media (VTM, Corning product number 25-500-CM, Corning, $\mathrm{NY}$ ) and tested in triplicate and in three independent runs.

Data analysis. Microsoft Excel was used to calculate the mean and variation of replicate $\mathrm{Ct}$ values, including standard deviation [SD (Ct)], coefficient of variation [\% CV (Ct)], and coefficient of variation based on linearized $\mathrm{Ct}$ values [\% Ct $\left(2^{-\mathrm{Ct}}\right)$ ]. Contingency $(2 \times 2)$ tables were analyzed in Microsoft Excel to determine the diagnostic sensitivity, diagnostic specificity, positive predictive value, and negative predictive value of the assays evaluated herein. Results provided by the originating laboratory were considered to be the reference standard for these calculations and comparisons of the assays evaluated here.

The degree of agreement between assay results was measured by Cohen's kappa using formulas from Watson and Petrie, 2010 [24]. The plot was generated with GraphPad Prism (version 9.0.0 for Windows, GraphPad Software, San Diego, California USA, www.graphpad.com). 


\section{Results}

Analytical sensitivity. The estimated LoD for the EZ-SARS-CoV-2 PCR assay was determined to be 250 copies per $\mathrm{ml}$ in VTM and AN sample matrix (equivalent to 1.75 copies per reaction) (Table 1). One replicate at 125 copies per $\mathrm{ml}$ was not detected with the AN matrix diluent. The LoD for the TaqPath COVID-19 Real-Time RT-PCR assay was estimated at 500 copies per $\mathrm{ml}$ (equivalent to 5 copies per reaction) (Table 1). The final LoD of the EZ-SARS-CoV-2 assay was determined testing 20 additional replicates containing 250 and 500 copies per ml. Nineteen of 20 replicates were positive at 250 copies per $\mathrm{ml}$ (Table 2) and the final LoD of the assay was determined to be 250 copies per $\mathrm{ml}$ which is equivalent to 1.75 genome copies per reaction.

Analytical specificity. The specificity of the primers and probes ( $\mathrm{N}$ gene assay 1 and 2 ) of the EZ-SARSCoV-2 RT-PCR were evaluated in silico and tested against a panel of 40 known human pathogens. In silico blast searches and alignment of the $\mathrm{N}$ gene 1 assay probe sequences revealed $92 \%$ sequence identity with SARS-CoV (AY345986.1), while the forward and reverse primers presented a lower sequence homology (70\% and $88 \%$, respectively) with SARS-CoV. The forward primer sequence of $\mathrm{N}$ gene assay 2 showed 100\% sequence identity to SARS-CoV (AY345986.1), whereas the $\mathrm{N}$ gene assay 2 reverse primer and probe sequences showed lower sequence homology to SARS-CoV ( $89 \%$ and $78 \%$, respectively). No significant homologies were observed between primer and probes of $\mathrm{N}$ gene assays 1 and 2 and the human genome, other coronaviruses or other common agents of the human microflora (Supplementary Table 1). Most importantly, testing of the panel of 40 known human pathogens did not result in cross amplification (Supplementary Table 2), confirming the specificity of the assay.

Assay reproducibility. The reproducibility of the EZ-SARS-CoV-2 assay was determined within a single test run (intra-run) and between independent runs (inter-run). Serial dilutions with known concentrations of the SARS-CoV-2 genome in VTM were tested in triplicate and in three independent PCR runs. As shown in Tables 3 and 4, the assay demonstrated high intra- and inter- run reproducibility. The standard deviation [SD (Ct)] of $\mathrm{Ct}$ values for replicate samples was less than 1.0 when tested on the ABI 7500 detection system. Only one set of replicates had a standard deviation over 1 (100 copies per $\mathrm{ml}$ on run 1) when tested using the QuantStudio 5 detection system. None of the coefficient of variation values based on $\mathrm{Ct}$ values [\%CV (Ct)] were greater than $3 \%$. Additionally, only two of the coefficient of variation values based on linearized $\mathrm{Ct}$ values [\% Ct $\left(2^{-\mathrm{Ct}}\right)$ ] exceeded $50 \%$ when tested on the $\mathrm{ABI} 7500$ system and none exceeded $50 \%$ when tested in the QuantStudio 5 detection system.

Additionally, intra-run repeatability of the EZ-SARS-CoV-2 RT-PCR assay was within expected parameters when SARS-CoV-2 genome copies were within the assay LoD, however, at the lower concentration tested (100 copies per $\mathrm{ml}$ ), greater variability was observed on the ABI 7500 platform with [SD (Ct)] greater than 1 , [\% CV (Ct)] greater than 3 , and [\% Ct $\left.\left(2^{-\mathrm{Ct}}\right)\right]$ greater than $50 \%$. Importantly, in both inter- and intra-run comparisons the replicates that demonstrated the greatest variability $(>50 \%)$ contained the lowest concentration of SARS-CoV-2 genomes/ml (100 copies per $\mathrm{ml}$ ), which is below the LoD of the assay (250 copies per $\mathrm{ml}$, Table 2). 
Clinical performance in individual upper respiratory samples. Clinical evaluation of the EZ-SARS-CoV-2 RT-PCR and comparison of its performance with the reference TaqPath ${ }^{\text {TM }}$ COVID-19 Real-Time RT-PCR assay or the Xpert $\circledast$ Xpress SARS-CoV-2 RT-PCR assays were performed on 201 NPS samples. Of these, 60 samples were originally tested on the Cepheid GeneXpert System using the Xpert ${ }^{\circledR}$ Xpress SARS-CoV2 RT-PCR assay (30 had SARS-CoV-2 positive test results and 30 had SARS-CoV-2 negative test results).

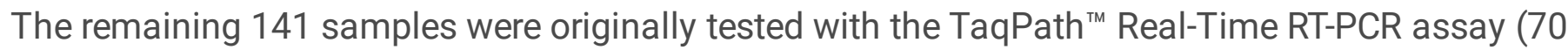
positive and 71 negative for SARS-CoV-2).

Among the 60 NPS samples previously tested using the Xpert ${ }^{\circledR}$ Xpress SARS-CoV-2 RT-PCR assay, the EZSARS-CoV-2 RT-PCR detected all 30 positive NPS specimens on both ABI 7500 and QuantStudio 5 platforms (Supplementary Table 3). Of the samples that were expected to be negative, two $(n=2)$ tested positive on the ABI 7500 platform and three $(n=3)$ were detected on the QuantStudio 5 platform (Supplementary Table 3). Two of the discrepant samples were detected by the EZ-SARS-CoV-2 RT-PCR assay on both platforms (ABI 7500 and QuantStudio 5).

When the set of 141 NPS samples originally tested with the TaqPath ${ }^{\text {TM }}$ COVID-19 Real-Time RT-PCR assay were tested with the EZ-SARS-CoV-2 RT-PCR, 66 or 64 of 70 positive NPS specimens were detected on the ABI 7500 or the QuantStudio 5 detection systems, respectively (Supplementary Table 4). Three of 6 discrepant samples were not detected on both platforms (ABI 7500 and QuantStudio 5). Of the NPS specimens that were expected to be negative, the EZ-SARS-CoV-2 RT-PCR assay detected 4 of these as positive on the $A B I 7500$ and 3 as positive on the QuantStudio 5 platform. One of the discrepant samples was positive on both platforms, the other 5 were only detected on a single platform.

The diagnostic sensitivity and specificity of the EZ-SARS-CoV-2 RT-PCR was estimated and compared to the TaqPath ${ }^{\text {TM }}$ COVID-19 Real-Time RT-PCR using test results from the 201 NPS samples included in our study (Table 5). Additionally, comparison of this dataset to the provided results was used to estimate the positive and negative predictive values of the EZ-SARS-CoV-2 RT-PCR assay. As shown in Table 6, the diagnostic sensitivity of the assay ranged between 94 - 96\% across the ABI 7500 and QuantStudio5 platforms, while the diagnostic specificity was $94.06 \%$. The positive predictive value (PPV) for the EZSARS-CoV-2 RT-PCR assay was $94 \%$ and the negative predictive value (NPV) ranged from 94 to $96 \%$.

Clinical performance in pooled upper respiratory samples. The performance of the EZ-SARS-CoV-2 assay and its feasibility for testing pooled respiratory samples was also evaluated. Ten individual positive NP swab samples with original Ct values greater than 28 were selected to create 10 pools. Three independent extractions and amplification runs were performed for each pool. Pools were assayed with both the EZSARS-CoV-2 RT-PCR assay and the TaqPath COVID-19 Real-Time RT-PCR assay.

To assess a pool size of 10, each of 10 pools comprised one SARS-CoV-2 positive NP swab sample and 9 negative NP swabs samples. The average diagnostic sensitivity on pools of 10 samples after three extraction and amplification runs were approximately $70 \%$ with the EZ-SARS-CoV-2 RT-PCR assay and approximately $50 \%$ with the TaqPath COVID-19 Combo Kit Multiplex Real-Time RT-PCR assay (Table 7). 
When a pool size of 5 was assessed, using the same 10 individual positive samples now combined with 4 negative NP samples, the average diagnostic sensitivity increased to $90 \%$ with the EZ-SARS-CoV-2 RTPCR assay and approximately 75\% with the TaqPath COVID-19 Combo Kit Multiplex Real-Time RT-PCR assay (Table 8).

Given the better performance of the assays on pools of 5 samples, additional pools of 5 were created with one positive and 4 negative samples each, including 10 more NP swab pools and 12 AN swab pools. These pools were extracted and amplified with the EZ-SARS-CoV-2 RT-PCR assay three times. The difference in $\mathrm{Ct}$ values between the individual positive sample and the corresponding pool of 5 is shown below (Table 9 for ABI 7500 platform, Table 10 for QuantStudio 5 platform).

Twelve pools of 5 SARS-CoV- 2 negative AN samples were generated at the same time as the SARS-CoV- 2 positive AN pools. No pools were detected after performing 3 extraction and RT-PCR assays on the ABI 7500 platform. On the QuantStudio 5 platform, only one pool (pool 4) was detected in one out of 3 runs, with a Ct value of 41.79 .

For pools of 5 contrived from one positive and four negative samples, the average Ct change observed for the entire study was 2.44 , with a standard deviation of 2.90. The expected Ct change for a 5 -fold dilution is approximately 2.3 cycles. The relationship between EZ-SARS-CoV-2 RT-PCR Ct values of the individual SARS-CoV-2 positive sample and in the corresponding pools of 5 is shown in Figure 1.

The change in Ct value between positive pools and corresponding individual positive specimens tested with the EZ-SARS-CoV-2 RT-PCR assay was also evaluated in additional pools. From 54 positive pools with Ct values ranging from 15.66 to 39.53 , the average $C t$ change was 2.84 cycles with a standard deviation of 1.94 .

Positive pooled samples were not detected 7 times out of 96 amplifications (32 pools extracted and assayed 3 times each), resulting approximately $93 \%$ diagnostic sensitivity for pools of 5 containing one SARS-CoV-2 positive sample.

\section{Discussion}

This study evaluated the clinical performance of the EZ-SARS-CoV-2 RT-PCR assay on individual and pooled human upper respiratory specimens. The analytical sensitivity of the EZ-SARS-CoV-2 RT-PCR assay was determined to be 250 copies/ml, which corresponds to approximately 1.75 genome copy equivalents per reaction. In comparison, the TaqPath COVID-19 RT-PCR assay was defined as 500 copies/ml herein ( 5 genomic copy equivalents per reaction), and is reported to be 10 genomic copy equivalents per reaction by the manufacturer [25]. The sensitivity of these RT-PCR assays is comparable to the US CDC RT-PCR panel for SARS-CoV-2, which is reported to be 5 RNA transcript copies/reaction [26]. In contrast, the cartridge-based Xpert Xpress SARS-CoV-2 Assay LoD was reported to be 250 copies/ml by the manufacturer in April 2020 but when the required $300 \mathrm{ml}$ sample input volume is considered, the LoD equates to 75 copies per reaction [27]. The specificity of the EZ-SARS-CoV-2 RT-PCR 
assay was assessed in silico and in vitro with a panel 40 bacterial, fungal, and viral human respiratory pathogens, including SARS and MERS coronaviruses. The EZ-SARS-CoV-2 RT-PCR assay did not amplify any pathogens other than SARS-CoV-2.

Both intra-assay and inter-assay variation of the EZ-SARS-CoV-2 RT-PCR assay were low. Importantly, in both inter- and intra-assay comparisons the replicates that demonstrated the greatest variability (CV (linearized $\mathrm{Ct}$ ) $>50 \%$ ) contained the lowest concentration of SARS-CoV-2 genomes/ml (100 copies per $\mathrm{ml})$, which is below the assay LoD (250 copies per $\mathrm{ml}$, Table 2 ).

The diagnostic sensitivity of the EZ-SARS-CoV-2 RT-PCR assay was compared to that of the Cepheid GeneXpert Xpress system using 60 NP samples. All 30 positive samples were correctly identified by both assays, however the EZ-SARS-CoV-2 RT-PCR assay identified 2 of the 30 negative samples as positive on both of 2 amplification platforms, with late positive Ct values. One of these was also identified as positive by the TaqPath COVID-19 RT-PCR assay on both amplification platforms. Given the differences in LoD, these samples may be true positives with low viral load. When compared to a larger set of 70 positives that were initially tested with the TaqPath COVID-19 RT-PCR assay elsewhere, 4 were not detected with the EZ-SARS-CoV-2 RT-PCR assay on the ABI 7500 Fast platform; 3 of these were not detected on the QuantStudio 5 platform as well. Given that these were archived samples, the possibility that shipping and/or freeze-thawing may have compromised the integrity of the viral RNA present in some of these samples cannot be formally excluded. Original false positive results in the initial test may also contribute to these discrepancies. Considering all 201 used in the present study, the diagnostic sensitivity of the EZSARS-CoV-2 RT-PCR assay was $94-96 \%$, with diagnostic specificity of $94 \%$. The high level of agreement is also represented by Cohen's kappa values of 0.92-0.93.

Testing the same set of 201 diagnostic samples allowed us to compare the performance of the EZ-SARSCoV-2 RT-PCR assay directly with the TaqPath COVID-19 RT-PCR assay as they were performed using the same nucleic acid elutions and on the same PCR instruments. The diagnostic sensitivity of the EZ-SARSCoV-2 RT-PCR assay was $94-96 \%$, higher than the 90\% determined for TaqPath COVID-19 RT-PCR assay on both platforms. However, the diagnostic specificity of the TaqPath COVID-19 RT-PCR assay was slightly higher than the EZ-SARS-CoV-2 RT-PCR assay (97-98\% versus $94 \%)$. The positive predictive value for the EZ-SARS-CoV-2 RT-PCR assay was approximately $94 \%$, whereas the negative predictive value was approximately 95\%. Overall, agreement between the EZ-SARS-CoV-2 and the TaqPath COVID-19 RT-PCR assays was high, supported by Cohen's kappa value of 0.93-0.94.

To increase our testing capacity and meet the high demand for surveillance testing at Cornell University, the feasibility of testing pooled samples was also investigated. Pooling one SARS-CoV-2 positive NP sample with 9 negative NP samples reduced the diagnostic sensitivity to 70\% with the EZ-SARS-CoV-2 assay and to $50 \%$ with the TaqPath COVID-19 kit. Pools of 5 samples yielded $93 \%$ diagnostic sensitivity and acceptable performance was found for both NP and AN swab specimens. Pooling is an important strategy to both increase the sample throughput and reduce the amount of reagents and supplies required. 
In summary, the EZ-SARS-CoV-2 RT-PCR assay has excellent performance using the automated extraction and amplification workflow with human upper respiratory samples. Results from this study support the use of the EZ-SARS-CoV-2 RT-PCR for pooled testing in upper respiratory human samples. Combining the high sensitivity of the EZ-SARS-CoV-2 assay with the ease of AN swab collection and ability to pool samples will facilitate a high throughput surveillance testing program. The slightly reduced sensitivity in pooled specimens can be compensated by high frequency sample collection, especially from those populations at higher risk of SARS-CoV-2 infection and spread.

\section{Declarations}

\section{Acknowledgments}

We thank the Office of the Vice Provost for Academic Integration for providing financial support for the validation studies described here. We would like to thank Drs. Lars Westblade and Melissa Cushing from Weill Cornell Medicine, Dr. Kiril Dimitrov from Texas Medical Veterinary Diagnostic Laboratory, and Dr. Kirsten St. George from Wadsworth Center, NYS Department of Health for providing archived clinical samples used for the validation study described here.

Funding This study was funded by the Cornell University Office of the Vice Provost for Academic Integration, Ithaca, New York, USA.

Conflicts of Interest The authors have no relevant financial or non-financial interests to disclose.

Availability of data Raw data is available upon request.

Code availability Not applicable

Authors' contributions The EZ-SARS-CoV-2 RT-PCR assay was designed by Rolf Rauh, Amy Saunders, and William M. Nelson. Samples were acquired by Elizabeth Plocharczyk and Diego G. Diel. Samples were processed and validation experiments were performed by Melissa Laverack, Roopa Venugopalan, Brittany Cronk, and XiuLin Zhang. Specificity experiments were performed by Rolf Rauh and Amy Saunders. Data was analyzed and the first manuscript was drafted by Rebecca L. Tallmadge and Diego G. Diel. All authors read and approved the final manuscript.

Ethics approval Not applicable

Consent to participate Not applicable

Consent for publication Not applicable

\section{References}


1. Bchetnia M, Girard C, Duchaine C, Laprise C (2020) The outbreak of the novel severe acute respiratory syndrome coronavirus 2 (SARS-CoV-2): A review of the current global status. J Infect Public Health 13:1601-1610. https://doi.org/10.1016/j.jiph.2020.07.011

2. Sohrabi C, Alsafi Z, O'Neill N, et al (2020) World Health Organization declares global emergency: A review of the 2019 novel coronavirus (COVID-19). Int J Surg 76:71-76.

https://doi.org/10.1016/j.ijsu.2020.02.034

3. World Health Organization Emergency Committee (2021) Statement on the sixth meeting of the International Health Regulations (2005) Emergency Committee regarding the coronavirus disease (COVID-19) pandemic. https://www.who.int/news/item/15-01-2021-statement-on-the-sixth-meetingof-the-international-health-regulations-(2005)-emergency-committee-regarding-the-coronavirusdisease-(covid-19)-pandemic. Accessed 2 Feb 2021

4. World Health Organization (2020) Considerations in the investigation of cases and clusters of COVID-19: interim guidance. https://www.who.int/publications/i/item/considerations-in-theinvestigation-of-cases-and-clusters-of-covid-19. Accessed 2 Feb 2021

5. Buitrago-Garcia D, Egli-Gany D, Counotte MJ, et al (2020) Occurrence and transmission potential of asymptomatic and presymptomatic SARSCoV-2 infections: A living systematic review and metaanalysis. PLoS Med 17:e100334. https://doi.org/10.1371/journal.pmed.1003346

6. Han D, Li R, Han Y, et al (2020) Covid-19: Insight into the asymptomatic sars-cov-2 infection and transmission. Int J Biol Sci 16:2803-2811. https://doi.org/10.7150/ijbs.48991

7. Oran DP, Topol EJ (2020) Prevalence of Asymptomatic SARS-CoV-2 Infection: A Narrative Review. Ann Intern Med 173:362-367. https://doi.org/10.7326/M20-3012

8. Arons MM, Hatfield KM, Reddy SC, et al (2020) Presymptomatic SARS-CoV-2 Infections and Transmission in a Skilled Nursing Facility. N Engl J Med 382:2081-2090. https://doi.org/10.1056/nejmoa2008457

9. Cheng HY, Jian SW, Liu DP, et al (2020) Contact Tracing Assessment of COVID-19 Transmission Dynamics in Taiwan and Risk at Different Exposure Periods before and after Symptom Onset. JAMA Intern Med 180:1156-1163. https://doi.org/10.1001/jamainternmed.2020.2020

10. Kimball A, Hatfield KM, Arons M, et al (2020) Asymptomatic and Presymptomatic SARS-CoV-2 Infections in Residents of a Long-Term Care Skilled Nursing Facility - King County, Washington, March 2020. MMWR Morb Mortal Wkly Rep 69:377-381. https://doi.org/10.15585/mmwr.mm6913e1

11. Wei WE, Li Z, Chiew CJ, et al (2020) Presymptomatic Transmission of SARS-CoV-2 - Singapore, January 23-March 16, 2020. MMWR Morb Mortal Wkly Rep 69:411-415. https://doi.org/10.15585/mmwr.mm6914e1

12. (2021) Supply Shortages Impacting COVID-19 and Non-COVID Testing. In: Am. Soc. Microbiol. https://asm.org/Articles/2020/September/Clinical-Microbiology-Supply-Shortage-Collecti-1. Accessed 3 Feb 2021 
13. Zhu N, Zhang D, Wang W, et al (2020) A Novel Coronavirus from Patients with Pneumonia in China, 2019. N Engl J Med 382:727-733. https://doi.org/10.1056/nejmoa2001017

14. King AMQ, Adams MJ, Carstens EB, Lefkowitz EJ (2012) Order - Nidovirales. In: Virus Taxonomy. Elsevier, New York, pp 784-794

15. Shabani E, Dowlatshahi S, Abdekhodaie MJ (2021) Laboratory detection methods for the human coronaviruses. Eur J Clin Microbiol Infect Dis 40:225-246. https://doi.org/10.1007/s10096-02004001-8

16. Cheng MP, Papenburg J, Desjardins M, et al (2020) Diagnostic Testing for Severe Acute Respiratory Syndrome-Related Coronavirus 2: A Narrative Review. Ann Intern Med 172:726-734. https://doi.org/10.7326/M20-1301

17. (2021) In Vitro Diagnostics EUAs | FDA. In: U.S. Food Drug Adm. website. https://www.fda.gov/medical-devices/coronavirus-disease-2019-covid-19-emergency-useauthorizations-medical-devices/vitro-diagnostics-euas. Accessed 8 Feb 2021

18. Dorfman R (1943) The Detection of Defective Members of Large Populations. Ann Math Stat 14:436-440. https://doi.org/10.1214/aoms/1177731363

19. Abdalhamid B, Bilder CR, McCutchen EL, et al (2020) Assessment of Specimen Pooling to Conserve SARS CoV-2 Testing Resources. Am J Clin Pathol 153:715-718. https://doi.org/10.1093/ajcp/aqaa064

20. Ben-Ami R, Klochendler A, Seidel M, et al (2020) Large-scale implementation of pooled RNA extraction and RT-PCR for SARS-CoV-2 detection. Clin Microbiol Infect 26:1248-1253. https://doi.org/10.1016/j.cmi.2020.06.009

21. Hirotsu Y, Maejima M, Shibusawa M, et al (2020) Pooling RT-qPCR testing for SARS-CoV-2 in 1000 individuals of healthy and infection-suspected patients. Sci Rep 10:18899. https://doi.org/10.1038/s41598-020-76043-z

22. Deka S, Kalita D (2020) Effectiveness of Sample Pooling Strategies for SARS-CoV-2 Mass Screening by RT-PCR: A Scoping Review. J Lab Physicians 12:212-218. https://doi.org/10.1055/s-00401721159

23. Needleman SB, Wunsch CD (1970) A general method applicable to the search for similarities in the amino acid sequence of two proteins. J Mol Biol 48:443-453. https://doi.org/10.1016/00222836(70)90057-4

24. Watson PF, Petrie A (2010) Method agreement analysis: A review of correct methodology. Theriogenology 73:1167-1179. https://doi.org/10.1016/j.theriogenology.2010.01.003

25. Thermo Fisher Scientific Inc. (2020) TaqPath COVID-19 Combo Kit and TaqPath COVID-19 Combo Kit Advanced Instructions for Use, Publication Number MAN0019181, Revision H.0

26. Lu X, Wang L, Sakthivel SK, et al (2020) US CDC real-time reverse transcription PCR panel for detection of severe acute respiratory syndrome Coronavirus 2. Emerg Infect Dis 26:1654-1665. https://doi.org/10.3201/eid2608.201246

27. Cepheid (2020) Xpert Xpress SARS-CoV-2 Assay ENGLISH Package Insert 302-3750 Revision C 


\section{Tables}

Table 1. Percent positive replicates of two-fold dilution series detected on the ABI 7500 platform.

\begin{tabular}{|lllllll|}
\hline & \multicolumn{7}{c|}{ Copies per ml $^{\mathbf{a}}$} \\
\hline Assay & Diluent & $\mathbf{1 , 0 0 0}$ & $\mathbf{5 0 0}$ & $\mathbf{2 5 0}$ & $\mathbf{1 2 5}$ & $\mathbf{6 2 . 5}$ \\
\hline EZ-SARS-CoV-2 PCR assay & VTM & 100 & 100 & 100 & 100 & $\mathbf{7 7 . 7 8}$ \\
\cline { 2 - 7 } & AN & 100 & 100 & 100 & 88.89 & 88.89 \\
\hline $\begin{array}{l}\text { TaqPath COVID-19 Real-Time RT-PCR assay S } \\
\text { gene }\end{array}$ & VTM & 100 & 100 & 100 & 77.78 & 77.78 \\
\cline { 2 - 7 } & AN & 100 & 100 & 100 & 55.56 & 33.33 \\
\hline $\begin{array}{l}\text { TaqPath COVID-19 Real-Time RT-PCR assay } \\
\text { ORF1ab }\end{array}$ & VTM & 100 & 100 & 77.78 & 100 & 55.56 \\
\cline { 2 - 7 } & AN & 100 & 100 & 100 & 100 & 22.22 \\
\hline $\begin{array}{l}\text { TaqPath COVID-19 Real-Time RT-PCR assay N } \\
\text { gene }\end{array}$ & VTM & 100 & 100 & 100 & 100 & 44.44 \\
\cline { 2 - 7 } & AN & 100 & 100 & 77.78 & 77.78 & 22.22 \\
\cline { 2 - 7 }
\end{tabular}

${ }^{\text {a}}$ Results represent percent of replicates detected in three independent runs.

Table 2. Final limit of detection (LoD) of EZ-SARS-CoV-2 Real-Time RT-PCR based on the analyses of twenty replicates at the 250 copies per $\mathrm{ml}$ in AN matrix on the ABI 7500 platform.

\begin{tabular}{|llllllll|}
\hline Replicate & Ct value & Replicate & Ct value & Replicate & Ct value & Replicate & Ct value \\
\hline 1 & 35.27 & 6 & 35.16 & 11 & 36.69 & 16 & 36.20 \\
\hline 2 & 36.20 & 7 & 35.48 & 12 & Undetermined & 17 & 37.29 \\
\hline 3 & 34.15 & 8 & 34.90 & 13 & 36.09 & 18 & 35.57 \\
\hline 4 & 34.54 & 9 & 35.13 & 14 & 35.71 & 19 & 37.60 \\
\hline 5 & 36.92 & 10 & 35.30 & 15 & 34.14 & 20 & 35.94 \\
\hline
\end{tabular}

Table 3. Intra-assay variation of EZ-SARS-CoV-2 Real-Time RT-PCR cycle threshold (Ct) values. 


\begin{tabular}{|c|c|c|c|c|c|c|}
\hline Plate & \multicolumn{2}{|c|}{ Copies per ml } & Mean (Ct) & $S D(C t)$ & $\% \mathrm{CV}(\mathrm{Ct})$ & $\% C V\left(2^{-C t}\right)$ \\
\hline \multicolumn{7}{|c|}{ ABI 7500 platform } \\
\hline 1 & \multicolumn{2}{|l|}{$10^{4}$} & 29.91 & 0.09 & 0.30 & 6.18 \\
\hline 1 & \multicolumn{2}{|l|}{$10^{3}$} & 33.56 & 0.05 & 0.16 & 3.78 \\
\hline 1 & \multicolumn{2}{|l|}{$10^{2}$} & 36.74 & 0.72 & 1.96 & 53.52 \\
\hline 2 & \multicolumn{2}{|l|}{$10^{4}$} & 29.86 & 0.03 & 0.10 & 2.09 \\
\hline 2 & \multicolumn{2}{|l|}{$10^{3}$} & 33.92 & 0.26 & 0.76 & 16.96 \\
\hline 2 & \multicolumn{2}{|l|}{$10^{2}$} & 38.07 & 0.83 & 2.19 & 62.83 \\
\hline 3 & \multicolumn{2}{|l|}{$10^{4}$} & 29.45 & 0.12 & 0.41 & 8.47 \\
\hline 3 & \multicolumn{2}{|l|}{$10^{3}$} & 32.94 & 0.37 & 1.14 & 26.08 \\
\hline 3 & \multicolumn{2}{|l|}{$10^{2}$} & 35.78 & 0.54 & 1.5 & 36.76 \\
\hline Quant & tudio & platform & & & & \\
\hline 1 & $10^{4}$ & 29.99 & 0.39 & 1.30 & & \\
\hline 1 & $10^{3}$ & 33.02 & 0.08 & 0.24 & & \\
\hline 1 & $10^{2}$ & 36.49 & 1.14 & 3.14 & & \\
\hline 2 & $10^{4}$ & 29.88 & 0.28 & 0.94 & & \\
\hline 2 & $10^{3}$ & 34.13 & 0.32 & 0.93 & & \\
\hline 2 & $10^{2}$ & 36.13 & 0.49 & 1.36 & & \\
\hline 3 & $10^{4}$ & 29.99 & 0.15 & 0.51 & & \\
\hline 3 & $10^{3}$ & 33.28 & 0.13 & 0.38 & & \\
\hline 3 & $10^{2}$ & 37.05 & 0.45 & 1.21 & & \\
\hline
\end{tabular}

Table 4. Inter-assay variation of EZ-SARS-CoV-2 RT-PCR cycle threshold (Ct) values. 


\begin{tabular}{|c|c|c|c|c|}
\hline Copies per ml & Mean (Ct) & $S D(C t)$ & $\% C V(C t)$ & $\% \operatorname{CV}\left(2^{-C t}\right)$ \\
\hline \multicolumn{5}{|c|}{ ABI 7500 platform } \\
\hline $10^{4}$ & 29.74 & 0.25 & 0.85 & 18.30 \\
\hline $10^{3}$ & 33.47 & 0.49 & 1.48 & 36.33 \\
\hline $10^{2}$ & 36.86 & 1.15 & 3.11 & 66.82 \\
\hline \multicolumn{5}{|c|}{ QuantStudio 5 platform } \\
\hline $10^{4}$ & 29.95 & 0.06 & 0.21 & 4.38 \\
\hline $10^{3}$ & 33.48 & 0.58 & 1.72 & 35.04 \\
\hline $10^{2}$ & 36.56 & 0.46 & 1.27 & 31.32 \\
\hline
\end{tabular}

Table 5. Comparison of EZ-SARS-CoV-2 RT-PCR assay (EZ) results with TaqPath ${ }^{\text {TM }}$ COVID-19 Combo Kit Multiplex Real-Time RT-PCR results for 201 NPS performed on the same elution in the Cornell COVID-19 Testing Laboratory. Results determined on the ABI 7500 platform are on the left and results determined by the QuantStudio 5 platform are on the right.

\begin{tabular}{|c|c|c|c|c|c|}
\hline$A B I 7500$ & $\begin{array}{l}\text { TaqPath } \\
\text { positive }\end{array}$ & $\begin{array}{l}\text { TaqPath } \\
\text { negative }\end{array}$ & $\begin{array}{l}\text { QuantStudio } \\
5\end{array}$ & $\begin{array}{l}\text { TaqPath } \\
\text { positive }\end{array}$ & $\begin{array}{l}\text { TaqPath } \\
\text { negative }\end{array}$ \\
\hline $\begin{array}{l}\text { EZ } \\
\text { positive }\end{array}$ & 92 & 10 & EZ positive & 91 & 9 \\
\hline $\begin{array}{l}\text { EZ } \\
\text { negative }\end{array}$ & 0 & 99 & EZ negative & 2 & 99 \\
\hline \multicolumn{2}{|c|}{ Diagnostic sensitivity } & 100.00 & \multicolumn{2}{|c|}{ Diagnostic sensitivity } & 97.85 \\
\hline \multicolumn{2}{|c|}{ Diagnostic specificity } & 90.83 & \multicolumn{2}{|c|}{ Diagnostic specificity } & 91.67 \\
\hline \multicolumn{2}{|c|}{ Positive predictive value } & 90.20 & \multicolumn{2}{|c|}{ Positive predictive value } & 91.00 \\
\hline \multicolumn{2}{|c|}{ Negative predictive value } & 100.00 & \multicolumn{2}{|c|}{ Negative predictive value } & 98.02 \\
\hline \multicolumn{2}{|c|}{$\begin{array}{l}\text { kappa }(95 \% \text { confidence } \\
\text { interval) }\end{array}$} & $\begin{array}{l}0.94(0.90- \\
0.97)\end{array}$ & \multicolumn{2}{|c|}{$\begin{array}{l}\text { kappa ( } 95 \% \text { confidence } \\
\text { interval) }\end{array}$} & $0.93(0.89-0.97)$ \\
\hline
\end{tabular}

Table 6. Comparison of EZ-SARS-CoV-2 RT-PCR assay (EZ) results with results for 201 NPS provided by other testing laboratories. Results determined on the $\mathrm{ABI} 7500$ platform are on the left and results determined by the QuantStudio 5 platform are on the right. 


\begin{tabular}{|llllll|}
\hline ABI 7500 & $\begin{array}{l}\text { Provided } \\
\text { positive }\end{array}$ & $\begin{array}{l}\text { Provided } \\
\text { negative }\end{array}$ & QuantStudio 5 & $\begin{array}{l}\text { Provided } \\
\text { positive }\end{array}$ & $\begin{array}{l}\text { Provided } \\
\text { negative }\end{array}$ \\
\hline EZ positive & 96 & 6 & EZ positive & 94 & 6 \\
\hline EZ negative & 4 & 95 & EZ negative & 6 & 95 \\
\hline Diagnostic sensitivity & 96.00 & Diagnostic sensitivity & 94.00 \\
\hline Diagnostic specificity & 94.06 & Diagnostic specificity & 94.06 \\
\hline Positive predictive value & 94.12 & Positive predictive value & 94.00 \\
\hline Negative predictive value & 95.96 & Negative predictive value & 94.06 \\
\hline $\begin{array}{l}\text { kappa (95\% confidence } \\
\text { interval) }\end{array}$ & $\begin{array}{l}0.93(0.89- \\
0.97)\end{array}$ & $\begin{array}{l}\text { kappa (95\% confidence } \\
\text { interval) }\end{array}$ & $\begin{array}{l}0.92(0.88- \\
0.96)\end{array}$ \\
\hline
\end{tabular}

Table 7. Diagnostic sensitivity with a pool size of 10 NP swabs; 10 pools tested.

\begin{tabular}{|lll|}
\hline Assay & Platform & Diagnostic Sensitivity \\
\hline EZ-SARS-CoV-2 RT-PCR & ABI 7500 & 70 \\
\hline & QuantStudio 5 & 73 \\
\hline TaqPath COVID-19 RT-PCR & ABI 7500 & 50 \\
& QuantStudio 5 & 47 \\
\hline
\end{tabular}

Table 8. Diagnostic sensitivity with a pool size of 5 NP swabs; 10 pools tested.

\begin{tabular}{|lll|}
\hline Assay & Platform & Diagnostic Sensitivity \\
\hline EZ-SARS-CoV-2 RT-PCR & ABI 7500 & 90 \\
\hline & QuantStudio 5 & 90 \\
\hline TaqPath COVID-19 RT-PCR & ABI 7500 & 77 \\
& QuantStudio 5 & 73 \\
\hline
\end{tabular}

Table 9. Results of EZ-SARS-CoV-2 RT-PCR with pool size of 5 on the ABI 7500 platform. 


\begin{tabular}{|c|c|c|c|c|}
\hline Pool & Specimen & Individual Ct value & Pooled Ct value ${ }^{a}$ & Ct change \\
\hline 1 & NP & 33.55 & 34.58 & 1.03 \\
\hline 2 & NP & 35.30 & 36.86 & 1.55 \\
\hline 3 & NP & 33.39 & 34.34 & 0.95 \\
\hline 4 & NP & 36.20 & 36.77 & 0.58 \\
\hline 5 & NP & 33.85 & 36.55 & 2.71 \\
\hline 6 & NP & 33.33 & 34.69 & 1.36 \\
\hline 7 & NP & 35.75 & 36.62 & 0.87 \\
\hline 8 & NP & 40.25 & Not detected & \\
\hline 9 & NP & 35.19 & 37.75 & 2.56 \\
\hline \multirow[t]{2}{*}{10} & NP & 35.93 & 37.51 & 1.58 \\
\hline & & \multicolumn{2}{|c|}{ Average (standard deviation) of Ct change: } & $1.47(0.74)$ \\
\hline 11 & NP & 30.83 & 33.94 & 3.10 \\
\hline 12 & NP & 28.97 & 31.79 & 2.81 \\
\hline 13 & NP & 33.41 & 32.40 & -1.01 \\
\hline 14 & NP & 32.86 & 36.19 & 3.33 \\
\hline 15 & NP & 33.95 & 36.49 & 2.54 \\
\hline 16 & NP & 31.77 & 33.50 & 1.72 \\
\hline 17 & NP & 32.00 & 31.16 & -0.85 \\
\hline 18 & NP & 28.24 & 30.28 & 2.03 \\
\hline 19 & NP & 30.16 & 33.28 & 3.11 \\
\hline \multirow[t]{2}{*}{20} & NP & 32.25 & 35.17 & 2.91 \\
\hline & & \multicolumn{2}{|c|}{ Average (standard deviation) of Ct change: } & $1.97(1.61)$ \\
\hline 21 & $A N^{b}$ & 19.02 & 18.15 & -0.87 \\
\hline 22 & AN & 19.86 & 18.90 & -0.96 \\
\hline 23 & AN & 28.50 & 27.09 & -1.41 \\
\hline 24 & AN & 31.64 & 38.62 & 6.98 \\
\hline
\end{tabular}




\begin{tabular}{|lllll|}
25 & AN & 31.75 & 34.71 & 2.96 \\
\hline 26 & AN & 32.96 & 37.70 & 4.74 \\
27 & AN & 33.33 & 37.22 & 3.89 \\
28 & AN & 25.50 & 29.87 & 4.37 \\
\hline 29 & AN & 34.00 & 39.12 & 5.12 \\
\hline 30 & AN & 22.00 & 24.93 & 2.93 \\
\hline 31 & AN & 23.75 & 25.93 & 2.18 \\
\hline 32 & AN & 32.25 & 34.76 & 2.51 \\
\hline & & Average (standard deviation) of Ct change: & $2.70(2.63)$ \\
\hline
\end{tabular}



${ }^{b}$ AN specimens and individual Ct values were kindly provided by the Wadsworth Center, NYS Department of Health. There was not enough volume for both individual and pooled extractions.

Table 10. Results of EZ-SARS-CoV-2 RT-PCR with pool size of 5 on the QuantStudio 5 platform for validation. 


\begin{tabular}{|c|c|c|c|c|}
\hline Pool & Specimen & Individual Ct value & Pooled Ct value & Ct change \\
\hline 1 & NP & 32.96 & 35.04 & 1.49 \\
\hline 2 & NP & 35.42 & 36.96 & 1.65 \\
\hline 3 & NP & 32.65 & 35.04 & 1.65 \\
\hline 4 & NP & 34.67 & 36.62 & 0.43 \\
\hline 5 & NP & 33.11 & 36.97 & 3.12 \\
\hline 6 & NP & 33.41 & 35.46 & 2.13 \\
\hline 7 & NP & 34.74 & 36.84 & 1.10 \\
\hline 8 & NP & 37.15 & 38.41 & -1.84 \\
\hline 9 & NP & 35.13 & 37.04 & 1.85 \\
\hline \multirow[t]{2}{*}{10} & NP & 35.72 & 38.29 & 2.36 \\
\hline & & \multicolumn{2}{|c|}{ Average (standard deviation) of Ct change: } & $2.17(0.70)$ \\
\hline 11 & NP & 33.00 & 33.88 & 0.88 \\
\hline 12 & NP & 31.60 & 31.67 & 0.08 \\
\hline 13 & NP & 35.53 & 32.24 & -3.29 \\
\hline 14 & NP & 34.79 & 36.05 & 1.26 \\
\hline 15 & NP & 34.12 & 36.24 & 2.12 \\
\hline 16 & NP & 32.28 & 33.53 & 1.25 \\
\hline 17 & NP & 32.24 & 31.48 & -0.76 \\
\hline 18 & NP & 28.76 & 30.35 & 1.59 \\
\hline 19 & NP & 30.87 & 33.00 & 2.12 \\
\hline \multirow[t]{2}{*}{20} & NP & 32.36 & 34.82 & 2.46 \\
\hline & & \multicolumn{2}{|c|}{ Average (standard deviation) of Ct change: } & $0.77(1.73)$ \\
\hline 21 & $A N^{b}$ & 19.02 & 17.98 & -1.04 \\
\hline 22 & AN & 19.86 & 18.73 & -1.13 \\
\hline 23 & AN & 28.50 & 26.97 & -1.53 \\
\hline 24 & AN & 31.64 & 38.44 & 6.80 \\
\hline
\end{tabular}




\begin{tabular}{|lllll|}
25 & AN & 31.75 & 35.01 & 3.26 \\
\hline 26 & AN & 32.96 & 37.95 & 4.99 \\
\hline 27 & AN & 33.33 & Not detected & \\
\hline 28 & AN & 25.50 & 29.92 & 4.42 \\
\hline 29 & AN & 34.00 & 36.82 & 2.82 \\
\hline 30 & AN & 22.00 & 24.89 & 2.89 \\
\hline 31 & AN & 23.75 & 25.98 & 2.23 \\
\hline 32 & AN & 32.25 & 34.53 & 2.28 \\
\hline & & Average (standard deviation) of Ct change: & $2.36(2.67)$ \\
\hline
\end{tabular}

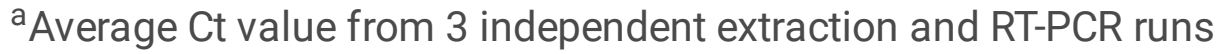

${ }^{\mathrm{b}} \mathrm{AN}$ specimens and individual Ct values were kindly provided by the Wadsworth Center, NYS Department of Health. There was not enough volume for both individual and pooled extractions.

\section{Figures}




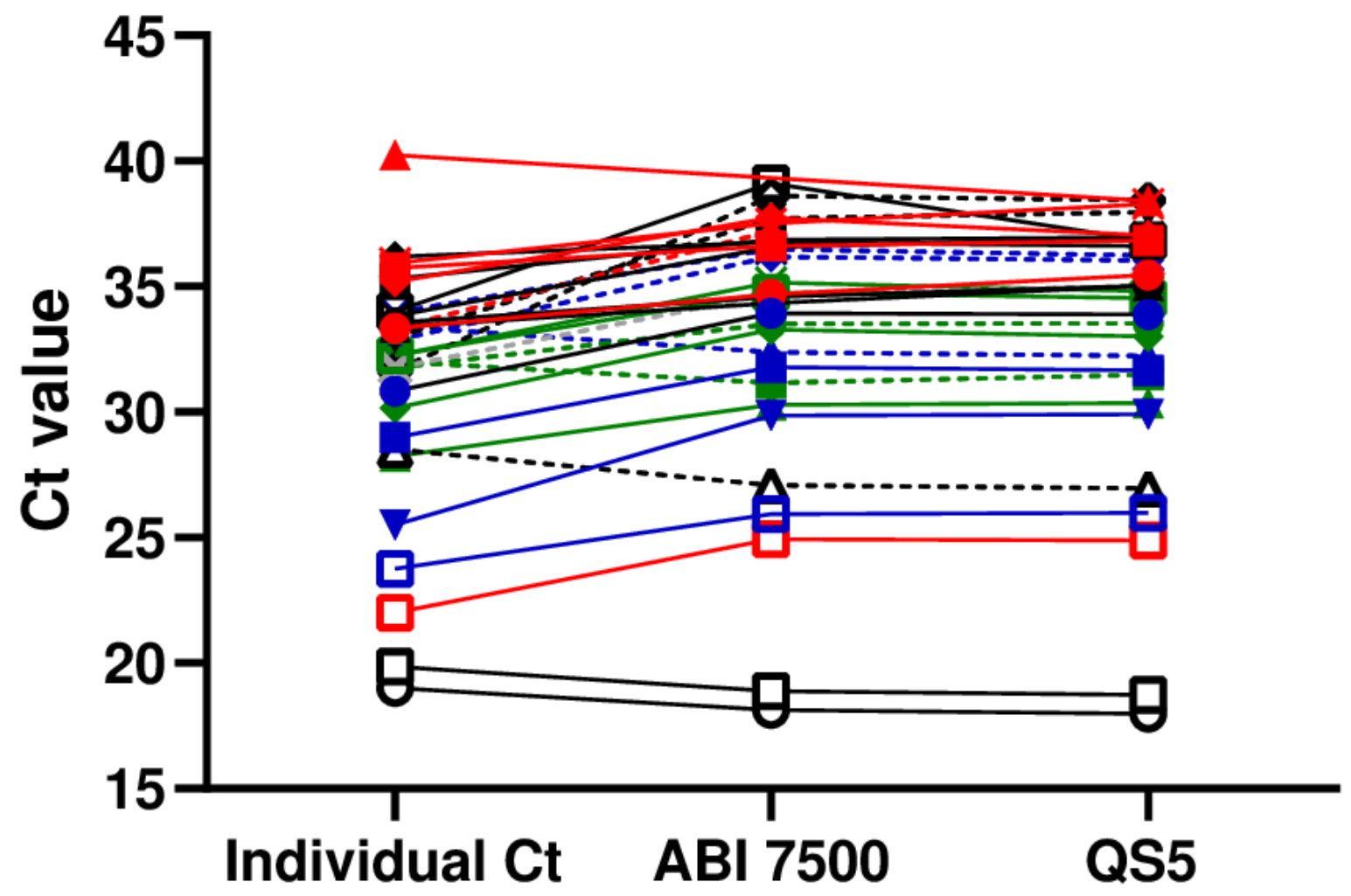

$\multimap$ Pool $1 \multimap$ Pool 9 -- Pool $17 \quad->-$ Pool 25

$\neg$ Pool $2 \rightarrow$ Pool $10 \rightarrow-$ Pool $18 \quad--\nabla--$ Pool 26

$\neg$ Pool $3 \multimap$ Pool $11 \multimap$ Pool $19 \multimap---\cdot$ Pool 27

$\neg$ Pool $4 \multimap$ Pool $12 \rightarrow$ Pool $20 \rightarrow$ Pool 28

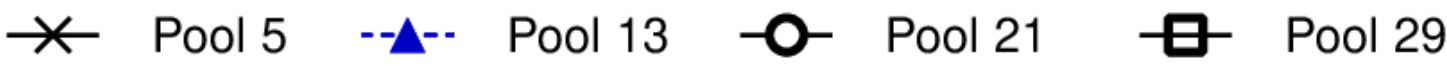



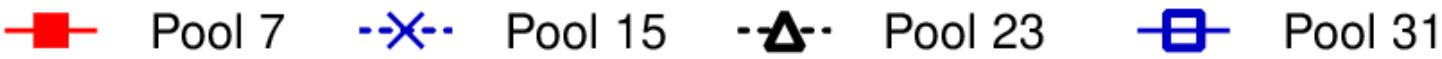

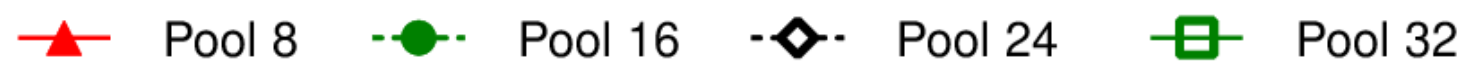

Figure 1

EZ-SARS-CoV-2 RT-PCR Ct values of individual and pooled samples. The Ct value of each individual SARS-CoV-2 positive sample is plotted and connected to the average pooled $\mathrm{Ct}$ value (Ct values from each of the three independent extraction and amplification runs averaged together) determined on either the $A B I 7500$ or QuantStudio 5 platform. 


\section{Supplementary Files}

This is a list of supplementary files associated with this preprint. Click to download.

- ValidationofSARSCoV2RTPCRSupplementaryTables.pdf 